\title{
Quantum folded string and integrability: from finite size effects to Konishi dimension
}

\author{
Nikolay Gromov, ${ }^{a, b}$ Didina Serban, ${ }^{c}$ Igor Shenderovich ${ }^{c, d}$ and Dmytro Volin ${ }^{e, f}$ \\ a Mathematics Department, King's College London, \\ The Strand, London WC2R 2LS, U.K. \\ ${ }^{b}$ St.Petersburg INP, Gatchina, 188 300, St.Petersburg, Russia \\ ${ }^{c}$ Institut de Physique Théorique, DSM, CEA, URA2306 CNRS, \\ Saclay, F-91191 Gif-sur-Yvette, France \\ ${ }^{d}$ St.Petersburg Department of Steklov Mathematical Institute RAS, \\ 27, Fontanka, 191023 St.Petersburg, Russia \\ ${ }^{e}$ Department of Physics, The Pennsylvania State University, \\ University Park, PA 16802, U.S.A. \\ ${ }^{f}$ Bogolyubov Institute for Theoretical Physics, \\ $14 b$ Metrolohichna str, Kyiv 03680, Ukraine \\ E-mail: nikolay.gromov@kcl.ac.uk, didina.serban@cea.fr, \\ shender.i@gmail.com, dvolin@psu.edu
}

ABSTRACT: Using the algebraic curve approach we one-loop quantize the folded string solution for the type IIB superstring in $A d S^{5} \times S_{5}$. We obtain an explicit result valid for arbitrary values of its Lorentz spin $S$ and R-charge $J$ in terms of integrals of elliptic functions. Then we consider the limit $S \sim J \sim 1$ and derive the leading three coefficients of strong coupling expansion of short operators. Notably, our result evaluated for the anomalous dimension of the Konishi state gives $2 \lambda^{1 / 4}-4+2 / \lambda^{1 / 4}$. This reproduces correctly the values predicted numerically in arXiv:0906.4240. Furthermore we compare our result using some new numerical data from the Y-system for another similar state. We also revisited some of the large $S$ computations using our methods. In particular, we derive finite-size corrections to the anomalous dimension of operators with small $J$ in this limit.

KEYwords: AdS-CFT Correspondence, Bethe Ansatz, Integrable Field Theories

ARXIV EPRINT: 1102.1040 


\section{Contents}

1 Introduction 1

2 Folded string quasi-classical quantization from Algebraic Curve 4

2.1 Classical solution 4

2.2 Off-shell fluctuations 6

2.3 One-loop shift 6

2.4 Short operator limit 8

3 The 1/ $\log S$ corrections for the long folded string 11

3.1 The one-loop corrections for the long string from algebraic curve 11

$\begin{array}{lll}3.2 & \text { The wrapping corrections from the Y-system } & 13\end{array}$

4 Conclusion $\quad 17$

$\begin{array}{ll}\text { A Simplified form of the one-loop integrals } & 17\end{array}$

\section{Introduction}

In the last decade, enormous progress was made in computing the spectrum of conformal dimensions of the $\mathcal{N}=4 \mathrm{SYM}$ theory in the planar limit, which by the AdS/CFT correspondence [1-3] is also the spectrum of strings moving in $A d S_{5} \times S^{5}$. This progress was made possible by the discovery of integrability [4-9] (also see review [10] for further references). The conjectured asymptotic Bethe ansatz equations [11-13] (also in the review cited above) interpolating between weak and strong coupling allowed to perform refined checks for operators with large charges. During the last few years it became clear that the exact solution for operators with finite charge is given by the Y-system [14-18]. At strong coupling the Y-system was successfully tested in the quasi-classical regime [19, 20]. The leading weak coupling correction from the Y-system [14] agrees with direct perturbative computations [21, 22]. The 5-loop corrections are equivalent [23-25] to the Lüscher corrections $[26,27]$ and also consistent [28-30] with the constraints from the BFKL equation [31].

In [14] the Y-system was combined with the vacuum TBA equations to produce an infinite set of integral equations for the $s l(2)$ part of the spectrum which were then solved numerically for the simplest nontrivial Konishi [32] operator. This method allowed to find the anomalous dimensions of short operators in a completely nonperturbative fashion starting from zero coupling and up to a relatively large value of the 't Hooft coupling $\lambda$. For extremely large $\lambda$ 's the numerical solutions become very slow, the largest value of $\lambda$ reached up to now is about 2000 [33]. It is however possible to extrapolate the numerical results 
and obtain the strong coupling expansion of the anomalous dimensions. The prediction obtained in [32] for the Konishi anomalous dimension $\gamma$ is

$$
\gamma+4=2.0004 \lambda^{1 / 4}+1.99 / \lambda^{1 / 4}
$$

The leading coefficient agrees with the prediction of [34] giving 2. This was also confirmed in a recent paper [35]. The sub-leading 2 was in disagreement with the calculation of [36] which appeared nearly the same time with [32]. In [37] it was discussed that this disagreement could be attributed to singularities which might change the integral equations at some large values of the coupling constant. In the present paper we confirm analytically the prediction (1.1) and solve this long standing discrepancy.

The strong coupling limit for the short operators looks very difficult to address both within the integrability and the perturbative string theory approaches. Although it is at least possible to define the set of equations to be solved using the Y-system, it seems rather hard to perform strong coupling expansion of these equations. At finite coupling the Yfunctions appear to have a complicated analytical structure [18], which can be already seen from its asymptotic solution [14]. It is likely that in general they have infinitely many cuts and in the strong coupling limit these cuts merge into each other.

On the string side, the semi-classical approach typically demands that the conserved charges scale as the coupling constant. For the case of the spinning folded string the two charges, the Lorentz spin $S$ and R-charge $J$, should scale in such a way that the ratios $\mathcal{S}=S / \sqrt{\lambda}, \mathcal{J}=J / \sqrt{\lambda}$ remain fixed. The expansion of the energy is of the form

$$
E \equiv \gamma+S+J=\sqrt{\lambda} E_{0}(\mathcal{S}, \mathcal{J})+E_{1}(\mathcal{S}, \mathcal{J})+\frac{1}{\sqrt{\lambda}} E_{2}(\mathcal{S}, \mathcal{J})+\ldots
$$

Then, in order to approach the short operator regime we re-expand the result in the limit when $S, J \sim 1$ and thus $\mathcal{S} \sim \mathcal{J} \ll 1$. As it was pointed out in [36] the expansion above reorganizes into a power series of the type

$$
E=\lambda^{1 / 4} a_{0}+\frac{1}{\lambda^{1 / 4}} a_{2}+\ldots,
$$

where only the classical energy $E_{0}$ contributes to the first coefficient $a_{0}$ and both $E_{1}$ and $E_{0}$ contribute to the coefficient $a_{2}$. Thus with some caution one may assume that the short strings with $S, J \sim 1$, which are in principle deeply quantum states, still can be reached using the quasi-classical methods. In this way, the complications with the direct treatment of the Y-system can be escaped. In this paper we compute the first two coefficients in the expansion (1.2) using the algebraic curve quantization procedure for an arbitrary $\mathcal{S}$ and $\mathcal{J}$ (see [38] for more details). What we found from our expressions for $E_{0}$ and $E_{1}$ is the following expansion

$$
E=\lambda^{1 / 4} \sqrt{2 n S}+\frac{1}{\lambda^{1 / 4}} \frac{2 J^{2}+S(3 S-2)}{4 \sqrt{2 n S}}+\ldots
$$

Notice that this procedure is very straightforward and is free from any ambiguity. What is important is that we do not get any logarithmic terms which would signal order-of-limits 
problems. From the ABA we know that the Konishi state in the $\operatorname{sl}(2)$ sector is given by $S=J=2$ and $n=1$. Substituting these values of the parameters we indeed obtain a result consistent with the prediction of [32] (1.1). In order to rule out an accidental coincidence of our result with that of [32] in the case of the Konishi operator, we also made a comparison of our result (1.4) with the numerical data obtained by [39] for another similar state, with $S=2 J=3$ and $n=1$.

The limit of short strings would not be the only case when the analytical continuation of the algebraic curve results gives reliable results in regions where the Lüscher corrections are large and the Y-system is difficult to handle analytically. Another example, which is treated in the second part of this paper, is that of the long strings with large Lorentz spin $S$ and small twist $J=\ell 4 g \log S$. It is well understood that this case can be obtained from the generic two-cut solution in the $s l(2)$ sector [40-43]

$$
E(\ell, \mathcal{S})=\left(\sqrt{\lambda} f_{0}(\ell)+f_{1}(\ell)+\frac{1}{\sqrt{\lambda}} f_{2}(\ell)\right) \log \mathcal{S}+\ldots
$$

The leading logarithmic scaling is a generic feature in gauge theories [44-47] and the coefficient of $\log \mathcal{S}$ is the so-called generalized scaling function. The functions $f_{0}(\ell), f_{1}(\ell)$, $f_{2}(\ell)$ were derived explicitly in $[40,40-43,48,49]$ respectively. It happens that all the three coefficients have a well-defined limit at $\ell=0$ and that in this limit they reproduce correctly the strong coupling expansion of the cusp anomalous dimension. In particular the $\ell=0$ result obtained in this order of limits coincides with the solution obtained [50-56] via the BES equation [13,57], which supposes $J=2$ and $S$ large and then $g \rightarrow \infty$. A recent review of this subject appeared in [58].

In this paper we also revisit the computation of the classical and one-loop energy for the long string both from the point of view of the algebraic curve and the Y-system, having in view the finite size corrections. We obtain results for all orders in $1 / \log S$ and we neglect terms of the order $\log S / S$ and higher. At $\ell=0$ the result is particularly simple ${ }^{1}$

$$
E_{\ell=0}=S+J+4 g\left(\log \frac{2 S}{g}-1\right)-\frac{3 \log 2}{\pi} \log \frac{2 S}{g}+\frac{6 \log 2}{\pi}+1-\frac{5 \pi}{12 \log (2 S / g)}+\mathcal{O}(1 / g)
$$

The sub-leading part in $\log S$ is the so-called virtual scaling function computed in [59,60] while the $1 / \log S$ part agrees with the results in [61-63]. In [63] the last term in (1.6) was given the simple interpretation of contribution of massless excitations propagating on a string of length $L=2 \log S$, with total result:

$$
\delta E_{1}=-\frac{\pi}{12 \log S} \times \text { (number of massless modes). }
$$

The massive modes lead to correction of the type $e^{-m L}$, where $m \sim \ell$ and $L=2 \log S$; these contributions have to be summed up properly in order to reproduce the massless limit.

In our computation, the four massive mode contributions come via the wrapping corrections. From the Y-system point of view, this part is constituted by two contributions

\footnotetext{
${ }^{1}$ Here we use the alternative notation $g=\sqrt{\lambda} / 4 \pi$.
} 
(virtual particle contribution and back-reaction of the roots) which become separately divergent when $\ell \rightarrow 0$. The algebraic curve computation does not see any divergence, and this may be compared to the particularly smooth behavior of the algebraic curve prediction for the short strings.

Finally, the contribution of the massless mode comes via the asymptotic Bethe ansatz. This might seem surprising, since at finite coupling there are no $1 / \log S$ corrections for the twist-two operator $J=2$, just the $(\log S)^{0}$ term [64,65]. This is obviously due to the different order of limits which are considered and might be explained by the fact that the bosonic modes of the $O(6)$ sigma model [66-68] acquire a dynamically generated mass at finite coupling. ${ }^{2}$

In conclusion, the algebraic curve method is a very reliable and efficient tool to obtain the one-loop results for a various range of string solution and seems to be free of some of the difficulties inherent to the direct treatment of the Y-system at strong coupling as well as from the ambiguities of the direct worldsheet quantization. The algebraic curve method may serve as a starting point to understand the behavior of the Y-system at higher loop order.

The plan of the paper is as follows. In section 2 we present the essential data for the algebraic curve which is necessary to derive the one-loop energy for the folded string at arbitrary $\mathcal{S}$ and $\mathcal{J}$. In subsection 2.4 we specialize to the operators with $S$ and $J$ finite and arbitrary $n$, including the Konishi operator, and we compare the result with the available numerical predictions. In section 3. we compute the spectrum for the long string to one loop, first from the algebraic curve and then from the Y-system.

\section{Folded string quasi-classical quantization from Algebraic Curve}

The algebraic curve method is one of the most advanced ways of computing the semiclassical corrections in the $A d S / C F T$ correspondence. The method is heavily based on integrability and is naturally free from the usual perturbation theory ambiguities. Some of these ambiguities are listed in appendix E of [70]. Some of the question raised there were recently explored in more detail in [71].

The method of the quasi-classical quantization was recently very pedagogically described in the recent AdS/CFT integrability review chapter [38] were more references can be found. We will adopt the same notations here.

\subsection{Classical solution}

The only input needed to proceed with the quantization is a set of quasi-momenta $\hat{p}_{i}, \tilde{p}_{i}, i=$ $1,2,3,4$ which constitute the algebraic curve. The folded string solution corresponds the curve with two symmetric cuts with real branch-points $\pm b, \pm a$ such that $1<a<b$. The explicit form of the quasi-momenta depends on the twist $J$ and the Lorentz spin $S$ can be

\footnotetext{
${ }^{2}$ We have been informed by B. Basso that this aspect will be investigated in [69].
} 
constructed using the methods of $[9,72,73]$. What one finds are the following expressions

$$
\begin{aligned}
p_{\hat{2}}= & \pi n-\frac{J}{2 g}\left(\frac{a}{a^{2}-1}-\frac{x}{x^{2}-1}\right) \sqrt{\frac{\left(a^{2}-1\right)\left(b^{2}-x^{2}\right)}{\left(b^{2}-1\right)\left(a^{2}-x^{2}\right)}} \\
& +\frac{2 a b S F_{1}(x)}{g(b-a)(a b+1)}+\frac{J(a-b) F_{2}(x)}{2 g \sqrt{\left(a^{2}-1\right)\left(b^{2}-1\right)}}, \\
p_{\tilde{2}}= & \frac{J x}{2 g\left(x^{2}-1\right)} .
\end{aligned}
$$

The integer $n$ (the mode number) is related to the number of spikes and $g=\frac{\sqrt{\lambda}}{4 \pi}$. All the other quasi-momenta can be found from the standard symmetry relations for the $\operatorname{sl}(2)$ sector

$$
\begin{aligned}
& p_{\hat{2}}(x)=-p_{\hat{3}}(x)=-p_{\hat{1}}(1 / x)=p_{\hat{4}}(1 / x), \\
& p_{\tilde{2}}(x)=-p_{\tilde{3}}(x)=p_{\tilde{1}}(x)=-p_{\tilde{4}}(x) .
\end{aligned}
$$

The functions $F_{1}(x)$ and $F_{2}(x)$ can be expressed in terms of the elliptic integrals:

$$
\begin{aligned}
& F_{1}(x)=i F\left(i \sinh ^{-1} \sqrt{\frac{(b-a)(a-x)}{(b+a)(a+x)}} \frac{(a+b)^{2}}{(a-b)^{2}}\right), \\
& F_{2}(x)=i E\left(i \sinh ^{-1} \sqrt{\frac{(b-a)(a-x)}{(b+a)(a+x)}} \frac{(a+b)^{2}}{(a-b)^{2}}\right) .
\end{aligned}
$$

The branch points $a, b$ are fixed once $S$ and $J$ are specified by the following equations

$$
\begin{aligned}
& S=2 n g \frac{a b+1}{a b}\left(b E\left(1-\frac{a^{2}}{b^{2}}\right)-a K\left(1-\frac{a^{2}}{b^{2}}\right)\right), \\
& J=\frac{4 n g}{b} K\left(1-\frac{a^{2}}{b^{2}}\right) \sqrt{\left(a^{2}-1\right)\left(b^{2}-1\right)} .
\end{aligned}
$$

Then the classical energy can be computed from

$$
\Delta=2 n g \frac{a b-1}{a b}\left(b E\left(1-\frac{a^{2}}{b^{2}}\right)+a K\left(1-\frac{a^{2}}{b^{2}}\right)\right) .
$$

In this way we get the classical energy of the generalized-folded solution as a function of $S$ and $J$.

It is important to notice that there is no question about identification of the operators corresponding to this classical solution in the framework of the algebraic curve. The algebraic curve was mapped to the Asymptotic Bethe Ansatz in [9, 74]. The super Yang-Mills operators at one loop are in one to one correspondence with the Bethe ansatz solutions up to a global bosonic symmetry action. Thus we can identify the class of operators we consider here. It is a subset of $\operatorname{tr}\left(D^{S} Z^{J}\right)+$ perm. Basically, since we use the algebraic curve formalism, we know automatically the whole set of conserved charges which provides an exhaustive information about the state. In particular this set includes the Konishi operator when $J=2, S=2$ and the mode number $n$ chosen to be $n=1$ as it follows from the one-loop spectrum at weak coupling. 


\subsection{Off-shell fluctuations}

An important feature of the algebraic curve quantization is that one can work with the off-shell fluctuation as it is described in detail in [38]. The off-shell fluctuation energies as functions of the spectral parameter $x$ are much simpler than the usual fluctuation energies, usually obtained in the world-sheet quantization procedure, which are functions of mode numbers. The former should coincide with the later when evaluated at the special points of the curve given by

$$
p_{i}\left(x_{k}^{i j}\right)-p_{j}\left(x_{k}^{i j}\right)=2 \pi k .
$$

In general one has to compute $8+8$ different off-shell energies corresponding to the number of the physical world-sheet degrees of freedom. However as it was shown in [75] in the rank one sectors one can express all of them in terms of just two, $\Omega^{\hat{2} \hat{3}}$ and $\Omega^{\tilde{2} \tilde{3}}$ :

$$
\begin{aligned}
& \Omega^{\hat{1} \hat{4}}(x)=-\Omega^{\hat{2} \hat{3}}(1 / x)-2, \\
& \Omega^{\hat{1} \hat{3}}(x)=\Omega^{\hat{2} \hat{4}}(x)=\frac{1}{2} \Omega^{\hat{1} \hat{4}}(x)-\frac{1}{2} \Omega^{\hat{1} \hat{4}}(1 / x)-1, \\
& \Omega^{\hat{1} \tilde{3}}(x)=\Omega^{\hat{1} \tilde{4}}(x)=\Omega^{\hat{4} \tilde{1}}(x)=\Omega^{\hat{4} \tilde{2}}(x)=\frac{1}{2} \Omega^{\tilde{2} \tilde{3}}(x)+\frac{1}{2} \Omega^{\hat{2} \hat{3}}(x), \\
& \Omega^{\hat{2} \tilde{3}}(x)=\Omega^{\hat{2} \tilde{4}}(x)=\Omega^{\hat{3} \tilde{1}}(x)=\Omega^{\hat{3} \tilde{2}}(x)=\frac{1}{2} \Omega^{\tilde{2} \tilde{3}}(x)-\frac{1}{2} \Omega^{\hat{2} \hat{3}}(1 / x)-1, \\
& \Omega^{\tilde{2} \tilde{3}}(x)=\Omega^{\tilde{2} \tilde{4}}(x)=\Omega^{\tilde{3} \tilde{1}}(x)=\Omega^{\tilde{3} \tilde{2}}(x)=\Omega^{\tilde{2} \tilde{3}}(x) .
\end{aligned}
$$

Since we are considering the $\operatorname{sl}(2)$ sector, the fluctuation energies in $S^{5}$ should be trivial and can be written down immediately:

$$
\Omega^{\tilde{2} \tilde{3}}(x)=+\frac{2}{a b-1} \frac{\sqrt{a^{2}-1} \sqrt{b^{2}-1}}{x^{2}-1} .
$$

Calculation of $\Omega^{\hat{2} \hat{3}}(x)$ is a little bit more involved. However the steps one should follow are exactly the same as in [75] and we simply give the result here

$$
\Omega^{\hat{2} \hat{3}}(x)=+\frac{2}{a b-1}\left(1-\frac{y(x)}{x^{2}-1}\right),
$$

where $y(x)=\sqrt{x-a} \sqrt{a+x} \sqrt{x-b} \sqrt{b+x}$.

For the analytical properties of these fluctuation energies see [75].

\subsection{One-loop shift}

In the previous sections we prepared all necessary ingredients needed for the one-loop corrections to the classical energy. As we mentioned in the previous section the usual excitation energies, typically used in the worldsheet calculations, can be obtained from the off-shell fluctuation energies $\Omega^{i j}(x)$ by setting $x$ to the value given be the equation (2.7) and then sum over all polarizations $(i j)$ and all mode numbers $k$. Doing this explicitly is almost impossible for the given quasi-momenta. The standard way to overcome this 
difficulty is to rewrite the sum as an integral (see, for example, [75]). For precise contour description see, for example, [76].

$$
\mathcal{E}=\frac{1}{2} \sum_{i j}(-1)^{F_{i j}} \oint \frac{d x}{2 \pi i}\left(\Omega^{i j}(x) \partial_{x} \log \sin \frac{p_{i}-p_{j}}{2}\right) .
$$

Here $F_{i j}$ is the fermionic number: $F_{i j}=0$ for bosonic polarizations and $F_{i j}=1$ for fermionic. The term $\partial_{x} \log \sin \frac{p_{i}-p_{j}}{2}$ has the poles at the solutions of (2.7). The contour of integration encircles all the possible fluctuations $x_{k}^{i j}$. This result is already explicit enough, however it is instructive to deform the contour into the unit circle (for each $(i j)$ ). During this contour deformation we can get two types of terms:

- Contribution from the integration on the unit circle for each polarization $(i j)$;

- Additional contribution from the cuts of the classical solution. Only the term with $(i j)=(\hat{2} \hat{3})$ gain such contribution.

It is also convenient to use the variable $z$ instead of $x$ :

$$
x=z+\sqrt{z^{2}-1}
$$

which maps the unit circle $|x|=1$ onto the interval $z \in[-1,1]$. Also we can split the logarithm in two parts:

$$
\log \sin \frac{p_{i}-p_{j}}{2}=\frac{i\left(p_{i}-p_{j}\right)}{2}+\log \left(1-e^{-i\left(p_{i}-p_{j}\right)}\right) .
$$

which holds up to some irrelevant constant. In this way we split the finite size effects from the asymptotic contribution. Indeed, for $z \in[-1,1] e^{-i\left(p_{i}-p_{j}\right)}$ is exponentially suppressed for large $J$. Substituting this into (2.11), we get two terms, $\delta E_{1}$ and $\delta E_{2}$ :

$$
\begin{aligned}
& \delta E_{1}=\sum_{i j}(-1)^{F_{i j}} \int_{-1}^{1} \frac{d z}{2 \pi i}\left(\Omega^{i j}(z) \partial_{z} \frac{i\left(p_{i}-p_{j}\right)}{2}\right) \\
& \delta E_{2}=\sum_{i j}(-1)^{F_{i j}} \int_{-1}^{1} \frac{d z}{2 \pi i}\left(\Omega^{i j}(z) \partial_{z} \log \left(1-e^{-i\left(p_{i}-p_{j}\right)}\right)\right) .
\end{aligned}
$$

One should take in account the contribution which we get by deforming the contour, which encircles the cuts $[-b,-a]$ and $[a, b]$. This contribution can be written as

$$
\delta E_{3}=-\frac{4}{a b-1} \int_{a}^{b} \frac{d x}{2 \pi i} \frac{y(x)}{x^{2}-1} \partial_{x} \log \sin p_{\hat{2}} .
$$

where we use $(2.2),(2.3)$. The one-loop shift is then given by

$$
E^{1-\text { loop }}=\delta E_{1}+\delta E_{2}+\delta E_{3}
$$

In appendix A we further expanding these integral using the relations between frequencies with different polarizations $(i j)$. 


\subsection{Short operator limit}

In this section we will exploit the explicit exact result for one-loop applicable for arbitrary $J, S \sim g$ which was derived in the previous section. These formulae involve a single integration and they can be evaluated numerically for various values of parameters.

The analytical evaluation of these integrals in general is not straightforward. In some limits, however, the integrands could simplify considerably so that the integration can be performed analytically. In this section we will consider one of such limits, namely we fix the ratio $r=J / S$ and then expand the result for small $S / g$. We will then motivate the relevance of this limit for the Konishi operator as well as for the similar type of operators with very few fields.

This limit is not completely trivial, the reason being that the algebraic curve becomes singular in this case: both positive branch points $a$ and $b$ approach the pole at $x=1$ as it can be easily seen from (2.4) and (2.5). We denote

$$
s \equiv \frac{\sqrt{2 S / n}}{\lambda^{1 / 4}}, r \equiv \frac{J}{S},
$$

where $\sqrt{\lambda}=4 \pi g$. In these notations the expansion of (2.4) and (2.5) gives

$$
\begin{aligned}
& a=1+\frac{r^{2}}{8} s^{3}+\frac{r^{2}-r^{4}}{128} s^{5}+\frac{r^{4}}{128} s^{6}-\frac{9 r^{2}+22 r^{4}-4 r^{6}}{4096} s^{7}+\ldots, \\
& b=1+2 s+2 s^{2}+\frac{7+r^{2}}{8} s^{3}-\frac{1-r^{2}}{4} s^{4}-\frac{85-34 r^{2}+2 r^{4}}{256} s^{5}+\ldots
\end{aligned}
$$

The classical energy gives

$$
\frac{\Delta}{n \sqrt{\lambda}}=s+\frac{3+2 r^{2}}{16} s^{3}-\frac{21-20 r^{2}+4 r^{4}}{512} s^{5}+O\left(s^{7}\right)+\ldots .
$$

We consider in some detail only the evaluation of $\delta E_{1}$ and then give the result for the others integrals. In general, the expression for $\delta E_{1}$ can be written conveniently as it is shown in appendix A:

$$
\delta E_{1}=-\frac{2}{\pi} \int_{0}^{1} \operatorname{Im}\left(\Omega^{\hat{2} \hat{3}}(z)-\Omega^{\tilde{2} \tilde{3}}(z)\right) \operatorname{Im}\left(p_{\hat{2}}^{\prime}(z)-p_{\tilde{2}}^{\prime}(z)\right) d z .
$$

First, assuming $z-1 \sim 1$ we expand the integrand to get

$$
-\int_{0}^{1} \frac{2 z^{2} s}{\left(z^{2}-1\right)^{2}} d z+\ldots
$$

Apparently the integral is divergent close to $z=1$. This divergence should be canceled when the integrand is treated more accurately for small $z-1$. There are two important scales when $z$ approaches 1: when one zooms close to the branch point $b$ which scales as $s^{2}$ then $z=1-s^{2} \zeta$ and when one further zooms so that we can distinguish the smallest branch point $a$ from 1 i.e. $z=1-s^{6} \xi$. For each of these scales the integral is divergent, however, when all the three regions are combined together the divergences must cancel. What we get for $\delta E_{1}$ is

$$
\delta E_{1} \simeq-s \log \frac{r s^{2}}{2}-\frac{s}{2} .
$$


The contributions $\delta E_{2}$ and $\delta E_{3}$ can be computed similarly. The results for these contributions are

$$
\begin{aligned}
& \delta E_{2} \simeq s \log s+c_{1} s \\
& \delta E_{3} \simeq s \log \frac{r s}{2}+\frac{s}{4}-c_{1} s
\end{aligned}
$$

where the constant $c_{1} \simeq 0.0203628454$. Notice that there are various log divergences which all cancel when the terms are combined together and the final result is very simple ${ }^{3}$

$$
\Delta^{1-\text { loop }}=\delta E_{1}+\delta E_{2}+\delta E_{3} \simeq-\frac{s}{4}=-\frac{\sqrt{2 S / n}}{4 \lambda^{1 / 4}} .
$$

In fact this result was previously obtained by us numerically with only two digits precision. This unpublished result was already used in [36] for the Konishi operator. The main difference with [36] is that we do not assume that $J=0$, instead from the point of view of algebraic curve and its relation to the ABA it is rather obvious that one should take $J=2$ for Konishi operator instead. Here we follow the approach of [36] with this small modification, which, however, changes the result considerably. ${ }^{4}$ Now we simply combine the classical energy (2.21) and the one-loop result (2.27) to get

$$
\Delta^{\text {classical }}+\Delta^{1-\text { loop }}=\lambda^{1 / 4} \sqrt{2 n S}+\frac{1}{\lambda^{1 / 4}} \frac{2 J^{2}+S(3 S-2)}{4 \sqrt{2 n S}} .
$$

The contribution to the first term comes solely from the classical energy, whereas both classical energy and the one-loop energy contribute to the second term. It is very tempting to assume that this pattern will continue further and in order to find the contribution to the next term one should also compute a two-loop correction. ${ }^{5}$

The equation (2.28) produces an infinite set of predictions which can be verified using the Y-system numerical approach proposed by [32].

For the Konishi state the prediction is already available [32]. To compare one should substitute $S=J=2$ and $n=1$ as we discussed above. Eq (2.28) produces

$$
\Delta^{\text {classical }}+\left.\Delta^{1-\text { loop }}\right|_{S=2, J=2, n=1}=2 \lambda^{1 / 4}+\frac{2}{\lambda^{1 / 4}}
$$

in the perfect agreement with the Y-system prediction of [32]. ${ }^{6}$

A natural question one can ask is whether this prediction is going to be correct for short operators other than Konishi. To address this question we consider an operator

\footnotetext{
${ }^{3}$ In order to get the ABA result with Hernandez-Lopez phase one should drop the $\delta E_{2}$ contribution. In this case one would get $-s \log s \simeq \frac{\sqrt{2 S / n}}{4 \lambda^{1 / 4}} \log \lambda$ divergence. Exactly this divergence was indeed observed in [77] for the Konishi anomalous dimension $(S=2, n=1)$ computed in the ABA framework.

${ }^{4}$ Almost simultaneously with us this point was also realized by A.Tseytlin according to our private communication.

${ }^{5}$ Strictly speaking this result holds assuming no non-perturbative terms contribute and that at each loop level the contribution can be represented as a regular series in $s$ vanishing at $s=0$.

${ }^{6}$ It is also compatible with the recent numerics with slightly higher precision [33].
} 


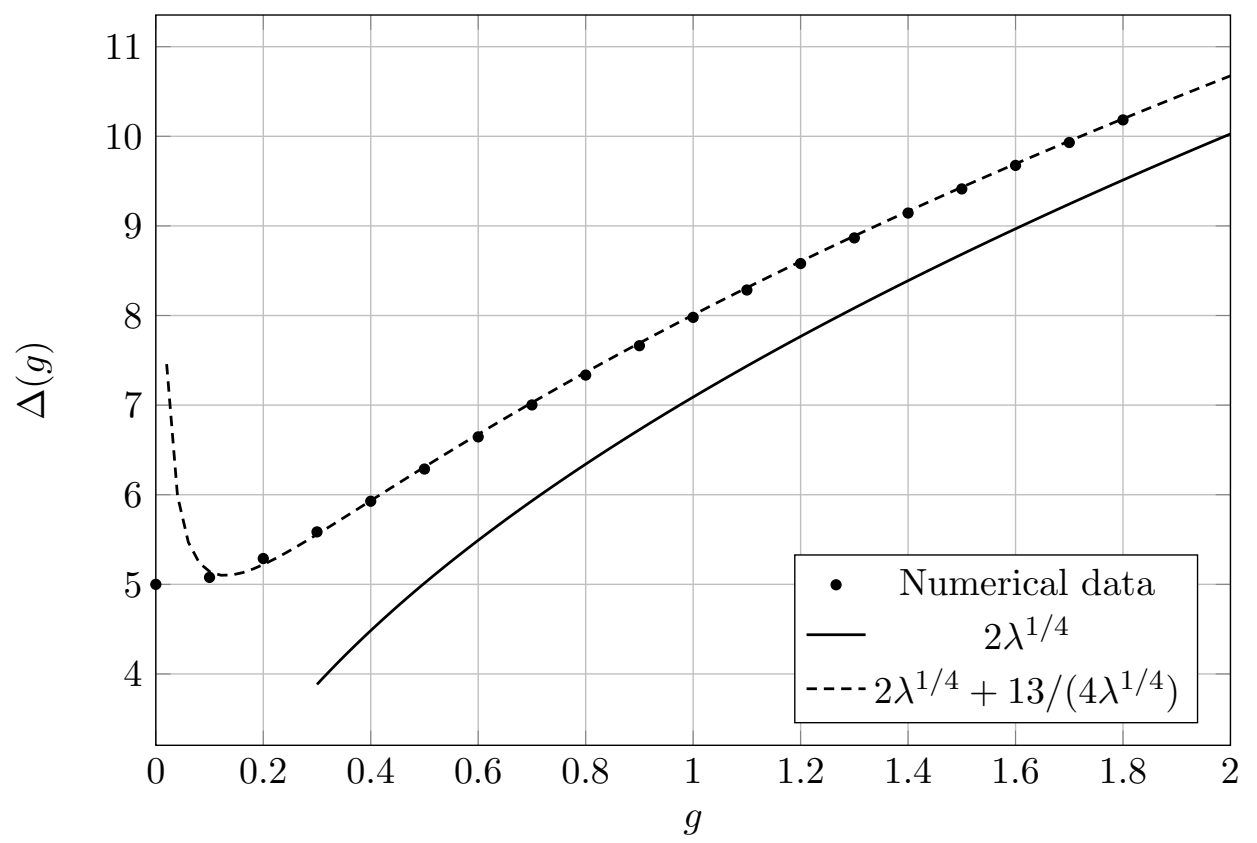

Figure 1. Numerical results from the Y-system for $J=3, S=2, n=1$ compared with our analytical strong coupling expansion (2.28). Here as everywhere in the paper $\lambda=16 \pi^{2} g^{2}$.

\begin{tabular}{|l|l|l|l|}
\hline$g$ & $\Delta$ & $g$ & $\Delta$ \\
\hline 0 & 5 & 0.9 & 7.6632 \\
0.1 & 5.0777 & 1.0 & 7.9794 \\
0.2 & 5.2883 & 1.1 & 8.2848 \\
0.3 & 5.5854 & 1.2 & 8.5801 \\
0.4 & 5.9275 & 1.3 & 8.8661 \\
0.5 & 6.2868 & 1.4 & 9.1436 \\
0.6 & 6.6456 & 1.5 & 9.4129 \\
0.7 & 7.0023 & 1.6 & 9.6752 \\
0.8 & 7.3354 & 1.7 & 9.9308 \\
\hline
\end{tabular}

Table 1. Konishi-like operator with $J=3$. The full dimension $\Delta=\gamma^{\text {anom }}+S+J$ for various values of $g$. Numerical data by [39] obtained with a new exact truncation method [78]. The numerical absolute error is about $\pm 3 \times 10^{-4}$.

similar to Konishi, with $J=3, S=2$ and $n=1$, which we denote as $(3,2,1)$. From (2.28) we see that our prediction produces

$$
\Delta^{(3,2,1)}=2 \lambda^{1 / 4}+\frac{13}{4 \lambda^{1 / 4}} .
$$

We compared this result with the preliminary numerical data shared with us by the authors of [39] (see table 1). As one can see from figure 1, our analytical results perfectly match these numerical points. ${ }^{7}$

\footnotetext{
${ }^{7}$ Let us also mention that our result (2.28) for $J=4, S=2, n=1$ gives $2 \lambda^{1 / 4}+5 / \lambda^{1 / 4}$ which seems to be
} 


\section{The $1 / \log S$ corrections for the long folded string}

In this section we derive the finite size corrections for the regime when $S$ is large and $J=$ $4 g \ell \log S$ with $\ell$ finite. The corrections are obtained by computing the three integrals (2.14)(2.16) (subsection 3.1) and in an alternative way by using the Y-system at one loop derived in [19] (subsection 3.2). We obtain the corrections at arbitrary order in $1 / \log S$, and we neglect all the inverse powers of $S$, as well as the $\log S / S$ terms. As a byproduct, we are re-deriving the known results for the generalized scaling function up to one loop [41, 42], as well as the virtual scaling function [59] to the same order. The computations are done for arbitrary $\ell$, but of course the formulas greatly simplify for the GKP [34] limit $\ell=0$. In this limit, the energy is given by

$$
E_{\ell=0}=S+J+4 g\left(\log \frac{2 S}{g}-1\right)-\frac{3 \log 2}{\pi} \log \frac{2 S}{g}+\frac{6 \log 2}{\pi}+1-\frac{5 \pi}{12 \log (2 S / g)}+\mathcal{O}(1 / g)
$$

The $(\log S)^{0}$ part is in agreement ${ }^{8}$ with $[59,60,62,81]$, while the $1 / \log S$ part agrees with the results in [61-63]. The $-5 \pi / 12 \log S$ term can be interpreted as coming from the finite size corrections associated to 5 massless bosonic fields $[63,69]$. At $\ell \neq 0$, four of these bosonic modes are massive, and their contribution is captured by the wrapping corrections. The fifth mode is massless, and it contributes via the anomaly term in the asymptotic Bethe ansatz equations. Although at weak coupling the asymptotic Bethe ansatz yields no $1 / \log S$ corrections for the twist-two operator $J=2$, [65], at strong coupling the situation is different. This can be attributed to the different order in which the limits $S \rightarrow \infty$ and $g \rightarrow \infty$ are taken.

\subsection{The one-loop corrections for the long string from algebraic curve}

In the limit of the long string $S \rightarrow \infty$, the endpoints $\pm b$ of the cuts of the curve go to infinity and the solution becomes effectively one-cut. The expression for the charges (2.4)-(2.6) simplify and, up to negative powers in $S$, we have

$$
\frac{S}{2 g}=b, \quad \frac{J}{4 g}=\sqrt{a^{2}-1} \log \frac{2 S}{a g}, \quad \frac{\Delta}{2 g}=\frac{S}{2 g}+a \log \frac{2 S}{a g} .
$$

In particular, we notice that the parameter $\ell \equiv J / 4 g \log S$ is related to the position of the endpoint $a$ of the cut by

$$
\ell=\sqrt{a^{2}-1}\left(1-\frac{\log (a g / 2)}{\log S}\right) .
$$

There will be trivial $1 / \log S$ terms coming from this relation between $\ell$ and $a$. After some manipulation, the elliptic functions reduce in the large spin limit to simpler functions and

\footnotetext{
consistent with some of the preliminary numerical results reported in [79] even though no solid statement was made there.

${ }^{8}$ The term $6 \log 2 / \pi+1$ was previously known by one of us, see the note added on pg. 24 in [80]. Please refer to the current paper for this coefficient.
} 
the quasi-momenta (2.1) become

$$
\begin{aligned}
& p_{\hat{2}}(x)=\frac{J}{2 g \sqrt{a^{2}-1}} \frac{x \sqrt{a^{2}-x^{2}}}{x^{2}-1}-4 \arctan \sqrt{\frac{a-x}{a+x}}, \\
& p_{\tilde{2}}(x)=\frac{J}{2 g} \frac{x}{x^{2}-1} .
\end{aligned}
$$

The off-shell frequencies (2.9) and (2.10) are given in this limit by

$$
\Omega^{\tilde{2} \tilde{3}}=\frac{2}{a} \frac{\sqrt{a^{2}-1}}{x^{2}-1}, \quad \Omega^{\hat{2} \hat{3}}=\frac{2}{a} \frac{\sqrt{a^{2}-x^{2}}}{x^{2}-1} .
$$

With these data in hand we are able to proceed to the computation of the three integrals giving the complete one-loop contribution to the energy. The easiest part to compute is $\delta E_{2}$, which can be reduced to

$$
\delta E_{2}=\frac{4 \sqrt{a^{2}-1}}{a \pi} \int_{1}^{\infty} d t \frac{\log \left(1-e^{-J t / 2 g}\right)}{\sqrt{1-1 / t^{2}}} \equiv \frac{\sqrt{a^{2}-1}}{a} \mathcal{I}(2 \ell \log S) .
$$

The following two representations of $\mathcal{I}(\alpha)$ are particularly useful:

$$
\begin{aligned}
\mathcal{I}(\alpha) & =-\sum_{n=1}^{\infty} \frac{4}{n \pi} K_{1}(n \alpha) \\
& =-\frac{2 \pi}{3 \alpha}+2+\frac{\alpha}{2 \pi}\left(2 \gamma_{E}-1+2 \log \left(\frac{\alpha}{4 \pi}\right)\right)+\frac{1}{\pi} \sum_{k=1}^{\infty} \frac{(-1)^{k} \zeta(2 k+1) \Gamma(2 k+1)}{\Gamma(k+1) \Gamma(k+2)(4 \pi)^{2 k}} \alpha^{2 k+1} .
\end{aligned}
$$

From the first representation ${ }^{9}$ we deduce that at large $\alpha, \mathcal{I}(\alpha) \sim e^{-\alpha} / \sqrt{\alpha}$, so for finite $\ell$ the associated finite-size corrections vanish exponentially. The second representation in (3.7) is useful in the small $\ell$ regime, where it gives ${ }^{10}$

$$
\delta E_{2} \simeq \frac{\ell \mathcal{I}(2 \ell \log S)}{\sqrt{1+\ell^{2}}}=-\frac{4 \pi}{12 \log S}+\mathcal{O}(\ell) .
$$

In the $O(6)$ language, [66, 67], this is the correction coming from four of five bosonic modes which are massive at finite $\mathcal{J}$ (hence exponential suppression at large $\alpha$ ) but become perturbatively massless in the limit $\mathcal{J} \rightarrow 0$.

The other two contributions to the one-loop energy are

$$
\begin{aligned}
& \delta E_{1}=-\frac{4}{a} \int_{U_{+}} \frac{d y}{2 \pi} \operatorname{Im} \frac{\sqrt{a^{2}-y^{2}}-\sqrt{a^{2}-1}}{y^{2}-1} \partial_{y} \operatorname{Im} G_{0}(y), \\
& \delta E_{3}=-\frac{4}{a} \int_{a}^{\infty} \frac{d y}{2 \pi} \frac{\sqrt{y^{2}-a^{2}}}{y^{2}-1} p^{\prime} \operatorname{coth} p,
\end{aligned}
$$

where we have denoted $p \equiv i p_{\hat{2}}(y+i 0), G_{0}(y) \equiv p_{\hat{2}}(y)-p_{\tilde{2}}(y)$ and the contour $U_{+}$is the upper half of the unit circle running clockwise. The last term can be split naturally into two parts

$$
\delta E_{3}=\delta E_{3, \text { an }}+\delta E_{3, \mathrm{~m}} .
$$

\footnotetext{
${ }^{9}$ A similar representation was obtained by B. Basso [69]

${ }^{10}$ Note that (3.8) appears only for $\ell \log S \ll 1$ which practically corresponds to $\mathcal{J} \rightarrow 0$ limit prior to the large $\mathcal{S}$ limit.
} 
The anomaly-like term $\delta E_{3 \text {,an }}$ contains the finite-size corrections associated to the fifth bosonic mode, which remains massless for arbitrary $\ell$

$$
\delta E_{3, \text { an }} \equiv-\frac{4}{a} \int_{a}^{\infty} \frac{d y}{2 \pi} \frac{\sqrt{y^{2}-a^{2}}}{y^{2}-1} p^{\prime}(\operatorname{coth} p-1)=-\frac{\pi}{12\left(1+\ell^{2}\right) \log S}+\mathcal{O}\left(1 / \log ^{2} S\right) .
$$

This is exactly the contribution of the only at $\mathcal{J} \neq 0$ massless mode identified by Giombi, Ricci, Roiban and Tseytlin [63]. A more refined evaluation of the anomaly part, up to $\log S / S$ terms, can be straightforwardly done using

$$
\delta E_{3, \mathrm{an}}=\sum_{n=1}^{\infty} f^{(n)}(0) \frac{\zeta(n+1)}{2^{n}}+\mathcal{O}(\log S / S) \quad \text { with } \quad f(p)=-\frac{2}{a \pi} \frac{\sqrt{y^{2}(p)-a^{2}}}{y^{2}(p)-1} .
$$

The remaining two contributions $\delta E_{1}$ and $\delta E_{3, \mathrm{~m}}$ reproduce the results already existing in the literature $[40-43,48]$ with

$$
\delta E_{3, \mathrm{~m}}=-\frac{4}{a} \int_{a}^{\infty} \frac{d y}{2 \pi} \frac{\sqrt{y^{2}-a^{2}}}{y^{2}-1} p^{\prime}=\frac{a-\left(a^{2}+1\right) \operatorname{arccoth} a}{a \pi} \log \frac{2 S}{a g}+\frac{4 \operatorname{arccoth} a}{a \pi}
$$

and

$$
\begin{aligned}
\delta E_{1}=- & \frac{4}{a} \int_{U_{+}} \frac{d y}{2 \pi} \operatorname{Im} \frac{\sqrt{a^{2}-y^{2}}-\sqrt{a^{2}-1}}{y^{2}-1} \partial_{y} \operatorname{Im} G_{0}(y) \\
=- & \frac{\left(a^{2}+1\right) \operatorname{arccoth} a^{2}+2 a^{2} \log \left(1-a^{-2}\right)+1}{a \pi} \log \frac{2 S}{a g} \\
& +\frac{1}{a \pi}\left(4 a \operatorname{arccot} a-4 \sqrt{a^{2}-1} \operatorname{arccot} \sqrt{a^{2}-1}+2 \log \left(1-a^{-4}\right)\right)
\end{aligned}
$$

At $\ell=0$ we get as expected

$$
\delta E_{1}+\delta E_{3, \mathrm{~m}}=-\frac{3 \log 2}{\pi} \log \frac{2 S}{g}+\frac{6 \log 2}{\pi}+1 .
$$

\subsection{The wrapping corrections from the $\mathrm{Y}$-system}

As it was shown in [19], exactly the same results which were obtained in the the previous section can be alternatively obtained directly from the Y-system, without reference to the algebraic curve. In this subsection we give such an alternative derivation. We find it instructive to identify the origin of different corrections from the point of view of the Ysystem, and this exercise may shed some light on the relation between the two approaches.

According to [19] the one-loop wrapping correction to the energy can be computed in terms of the following object

$$
\mathcal{M}_{0}=\log \frac{(f \Delta-1)^{4}(\bar{f} \Delta-1)^{4}}{(\Delta-1)^{4}(f \bar{f} \Delta-1)^{2}\left(f^{2} \Delta-1\right)\left(\bar{f}^{2} \Delta-1\right)}
$$

where

$$
f(z)=\exp \left(-i G(x(z)), \quad \bar{f}(z)=\exp \left(+i G(1 / x(z)), \quad \Delta=\exp \left(-\frac{J}{2 g \sqrt{1-z^{2}}}\right)\right.\right.
$$


and $G(x)$ is the resolvent

$$
G(x)=\frac{1}{g} \sum_{j=1}^{S} \frac{1}{x-x_{j}} \frac{x_{j}^{2}}{x_{j}^{2}-1} .
$$

The expression of the energy at one loop, including the finite-size correction, is

$$
E=\sum_{j=1}^{S} \frac{x_{j}^{2}+1}{x_{j}^{2}-1}+\int_{-1}^{1} \frac{d z}{2 \pi} \frac{z}{\sqrt{1-z^{2}}} \partial_{z} \mathcal{M}_{0}=\sum_{j=1}^{S} \frac{x_{j}^{2}+1}{x_{j}^{2}-1}-\int_{-1}^{1} \frac{d z}{2 \pi} \frac{1}{\left(1-z^{2}\right)^{3 / 2}} \mathcal{M}_{0} .
$$

The integration is done in the mirror regime, with $x(z)=z+i \sqrt{1-z^{2}}$. The second term in (3.19) is given by the contribution of the virtual particles circulating along the circumference of the system and which scatter with the magnons with rapidity $x_{j}$. We are therefore going to call this term the virtual particle contribution. In finite volume, the positions of the Bethe roots $x_{j}$ are slightly shifted from their infinite volume positions due to their interaction with the virtual particles; we are going to call this effect backreaction. In the one-loop limit, the backreaction can be taken into account [19] by adding an extra potential term to the Bethe ansatz equations, which become

$$
\begin{aligned}
2 \pi n= & p(x+i 0)+p(x-i 0)+\alpha(x) p^{\prime}(x) \cot p(x)+\mathcal{V}(x) \\
& -2 i \sum_{k=1}^{M} \int_{-1}^{1} d z\left(r(x, z) \mathcal{M}_{+}-r(1 / x, z) \mathcal{M}_{-}+u(x, z) \mathcal{M}_{0}\right)
\end{aligned}
$$

with

$$
p(x)=\frac{J}{2 g} \frac{x}{x^{2}-1}+G(x) \quad \text { and } \quad \alpha(x)=\frac{1}{g} \frac{x^{2}}{x^{2}-1} .
$$

The effective potential in the second line of (3.20) is given in term of the kernels

$$
r(x, z)=\frac{x}{x^{2}-1} \frac{\partial_{z}}{2 \pi g} \frac{1}{x-x(z)}, \quad u(x, z)=\frac{x}{x^{2}-1} \frac{\partial_{z}}{2 \pi g} \frac{1}{x^{2}(z)-1},
$$

and the functions

$$
\mathcal{M}_{+}=\log \frac{(f \Delta-1)^{2}}{\left(f^{2} \Delta-1\right)(f \bar{f} \Delta-1)}, \quad \mathcal{M}_{-}=\log \frac{(\bar{f} \Delta-1)^{2}}{\left(\bar{f}^{2} \Delta-1\right)(f \bar{f} \Delta-1)} .
$$

By inspection, the imaginary part of the resolvent $G(x)$ in the mirror regime is always negative with $\operatorname{Im} G(x) \sim-\log S$, so that we have

$$
\mathcal{M}_{0} \simeq-4 \log (1-\Delta)-\Delta\left(f^{2}+\bar{f}^{2}+2 f \bar{f}-4 f-4 \bar{f}\right)=-4 \log (1-\Delta)-4 \Delta R(R-2),
$$

where $R=\exp (\operatorname{Im} G(x)) \cos (\operatorname{Re} G(x))$. The last term in $\mathcal{M}_{0}$ is suppressed by a negative power of $S$. The only region where $R$ can be close to 1 is $x \simeq 1$, but in this region it is $\Delta$ which is exponentially suppressed. We conclude that the correction to the energy due to the virtual particles is

$$
\delta E_{v}=4 \int_{-1}^{1} \frac{d z}{2 \pi} \frac{1}{\left(1-z^{2}\right)^{3 / 2}} \log (1-\Delta)=\mathcal{I}(2 \ell \log S) .
$$


with $\mathcal{I}(\alpha)$ defined in (3.6) and (3.7). It is interesting to note that the virtual particle correction is singular when $\ell \rightarrow 0$, and that this divergence will be compensated by the backreaction of the roots. It is likely that such a phenomenon happens whenever the endpoint $a$ of the cut approaches the singularity $x=1$. In particular (logarithmic) singularities appear for separate $E_{i}$ terms in the small $\mathcal{S}, \mathcal{J}$ limit, see section 2.4. A similar effect is observed when $f_{2}(\ell)$ (see (1.5)) is expanded at small $\ell$ [43]. This partially reflects the complicated analytical structure of the Y-system.

Let us now compute the backreaction term, i.e. the second line in the BES equation (3.20). The contribution from $\mathcal{M}_{ \pm}$is vanishing again as a negative power of $S$. The term containing $\mathcal{M}_{0}$ is simply

$$
8 i \frac{x}{x^{2}-1} \int_{-1}^{1} \frac{d z}{2 \pi g} \partial_{z}\left(\frac{1}{x(z)^{2}-1}\right) \log (1-\Delta)=\frac{\mathcal{I}(\alpha)}{g} \frac{x}{x^{2}-1} .
$$

Let us remind that at the leading order the asymptotic Bethe ansatz equations are written as

$$
2 \pi n=G_{0}(x+i 0)+G_{0}(x-i 0)+2 V_{0}(x)
$$

with

$$
2 V_{0}(x)=\frac{J}{g} \frac{x}{x^{2}-1}=4 \ell \log S \frac{x}{x^{2}-1}
$$

and $G_{0}(x)$ a function analytic everywhere except of the cuts on the intervals $(-\infty,-a) \cup$ $(a, \infty)$. We conclude that the only effect of the backreaction at one loop is to renormalize the coefficient of the potential term and therefore to renormalize $\ell$ by a one-loop quantity

$$
\ell \rightarrow \tilde{\ell}=\ell+\frac{\mathcal{I}(\alpha)}{4 g \log S} \simeq \ell-\frac{\pi}{12 \ell g \log ^{2} S} .
$$

To fix uniquely the solution of the leading order equation (3.27) we have to supply the asymptotics at infinity for $G_{0}(x)$. This is a little bit tricky if we have sent the endpoints of the two cuts $(-b,-a)$ and $(a, b)$ to infinity first. A straightforward procedure is to solve the equation (3.27) for finite $b$ (see [41]), imposing that $G_{0}(x) \sim 1 / x$ at $x \rightarrow \infty$ and then take the limit of infinite $b$. The result of this procedure would give $G_{0}(x)=p_{\hat{2}}(x)-p_{\tilde{2}}(x)$ with $p_{\hat{2}}(x), p_{\tilde{2}}(x)$ from (2.1). An alternative is to work directly with $b \rightarrow \infty$ and impose the same asymptotics for $G_{0}(x)$ as in the weak-coupling, one loop limit [82]

$$
G_{0}(x) \sim 2 i \log (S / g x) \quad \text { for } \quad x \rightarrow \infty-i 0 .
$$

The solution to the equation (3.27) supplemented with this condition at infinity reads

$$
\begin{aligned}
G_{0}(x) & =\sqrt{a^{2}-x^{2}} \oint_{\mathcal{C}} \frac{d y}{2 \pi i} \frac{V_{0}(y)}{(x-y) \sqrt{a^{2}-y^{2}}}-4 \arctan \sqrt{\frac{a-x}{a+x}} \\
& =\frac{J}{2 g} \frac{x}{x^{2}-1}\left(\frac{\sqrt{a^{2}-x^{2}}}{\sqrt{a^{2}-1}}-1\right)-4 \arctan \sqrt{\frac{a-x}{a+x}}
\end{aligned}
$$


where in the first line the contour of integration $\mathcal{C}$ encircles the cuts $(-\infty,-a) \cup(a, \infty)$ and can be closed at infinity counterclockwise. The value of $a$ is fixed by the asymptotics at infinity (3.30) and it yields the same condition as (3.3)

$$
\sqrt{a^{2}-1}=\frac{J}{4 g \log (2 S / a g)}=\ell+\mathcal{O}(1 / \log S) .
$$

The anomalous dimension at leading order is given by

$$
\begin{aligned}
E_{0} & =-\oint_{\mathcal{C}} \frac{d x}{2 \pi i} \frac{2}{x^{2}-1} \frac{G_{0}(x)}{\alpha(x)}=\frac{J}{\sqrt{a^{2}-1}}\left(a-\sqrt{a^{2}-1}\right)-\frac{4 g}{a} \\
& =4 g \log S\left(\sqrt{1+\ell^{2}}-\ell\right)+\mathcal{O}\left((\log S)^{0}\right)
\end{aligned}
$$

with the integration contour running counterclockwise around $x=0$. Now we can estimate the one-loop correction from the backreaction due to the shift $\ell \rightarrow \ell+\delta \ell$ from equation (3.29),

$$
\delta E_{b}=4 g \log S \delta\left(\sqrt{1+\ell^{2}}-\ell\right)=-\mathcal{I}(2 \ell \log S)+\frac{\ell \mathcal{I}(2 \ell \log S)}{\sqrt{1+\ell^{2}}}
$$

The one-loop wrapping corrections are then given by:

$$
\delta E_{w}=\delta E_{v}+\delta E_{b}=\frac{\ell \mathcal{I}(2 \ell \log S)}{\sqrt{1+\ell^{2}}}=-\frac{4 \pi}{12 \log S}+\mathcal{O}((\ell \log S) \log (\ell \log S))
$$

and they coincide with the contribution of the four massive modes $\delta E_{2}(3.8)$.

The wrapping corrections give the $1 / \log S$ corrections corresponding to only four of the five bosonic modes. To find the fifth one we are going to solve the one-loop equation for the resolvent

$$
0=G_{1}(x+i 0)+G_{1}(x-i 0)+2 V_{1}(x)
$$

with

$$
\frac{2 V_{1}(x)}{g}=\mathcal{V}(x)+\alpha(x) p_{0}^{\prime} \cot p_{0}, \quad p_{0}(x)=G_{0}(x)+V_{0}(x) .
$$

Here $\alpha(x) p_{0}^{\prime} \cot p_{0}$ is the so-called anomaly term and $\mathcal{V}(x)$ is the Hernandez-Lopez phase with integral representation [76]

$$
\mathcal{V}(x)=\int_{U^{+}} \frac{d y}{2 \pi}\left(\frac{\alpha(x)}{x-y}-\frac{\alpha(1 / x)}{1 / x-y}\right) \partial_{y}\left(G_{0}(y)-G_{0}(1 / y)\right),
$$

where the integral is taken clockwise on the upper half of the unit circle $U_{+}$. The solution to the one-loop equation can be again written in an integral form [41, 42]

$$
G_{1}(x)=\oint_{\mathcal{C}} \frac{d y}{2 \pi i} \frac{V_{1}(y)}{(x-y)} \frac{\sqrt{a^{2}-y^{2}}}{\sqrt{a^{2}-x^{2}}} .
$$

The one-loop correction to the energy is given, similarly to the leading order, by

$$
E_{1, \mathrm{ABA}}=-\oint_{\mathcal{C}} \frac{d x}{2 \pi i} \frac{2 G_{1}(x)}{x^{2}}=\frac{2}{a} \oint_{\mathcal{C}} \frac{d y}{2 \pi i} \frac{\sqrt{a^{2}-y^{2}}}{y^{2}} V_{1}(y)
$$


Substituting the value of the potential $V_{1}(x)$ we retrieve the contributions (3.9) from the algebraic curve computation

$$
\begin{aligned}
E_{1, \mathrm{ABA}} & =-\frac{4}{a} \int_{a}^{\infty} \frac{d y}{2 \pi} \frac{\sqrt{y^{2}-a^{2}}}{y^{2}-1} p^{\prime} \operatorname{coth} p-\frac{4}{a} \int_{U_{+}} \frac{d y}{2 \pi} \operatorname{Im} \frac{\sqrt{a^{2}-y^{2}}-\sqrt{a^{2}-1}}{y^{2}-1} \partial_{y} \operatorname{Im} G_{0}(y) \\
& =\delta E_{1}+\delta E_{3} .
\end{aligned}
$$

This result confirms that the asymptotic Bethe ansatz contribution is captured by $\delta E_{1}+\delta E_{3}$ and that the wrapping corrections are reproduced by $\delta E_{2}$.

\section{Conclusion}

We have computed one-loop correction to the energy of the folded string in the limit of large angular momentum in $A d S_{5}$ and on the sphere, by using the algebraic curve method and alternatively the Y-system method. By extrapolating the results at small angular momenta, we obtain predictions for the first few coefficients in the strong coupling expansion. The result confirms the numerical prediction from the Y-system for the Konishi operator [32] and more recent numerical results for similar operators [39]. In the limit of large Lorentz spin and small R-charge, we have computed the corrections to the logarithmic scaling and in particular we have reproduced the $1 / \log S$ corrections obtained in [61-63].

\section{Acknowledgments}

We would like to thank Benjamin Basso, Vladimir Kazakov, Gregory Korchemsky, Ivan Kostov, Sebastien Leurent, Radu Roiban, and Arkady Tseytlin for interesting discussions. The work of D.V. was supported by the US Department of Energy under contracts DEFG02-201390ER40577. I.S. was partially supported by the grant RFFI 11-01-00570. N.G. was partially supported by the grant RFFI 06-02-16786.

Note Added. While we were preparing these results for publication we became aware that the authors of [36] also found a way to correct their previous result concerning the Konishi dimension. This will be published in [83]. A different approach to compute analytically the Konishi dimension in the pure spinor formalism was developed in [84].

\section{A Simplified form of the one-loop integrals}

Using symmetry relations (2.2), (2.3) (2.8), one can rewrite sums $\delta E_{1}$ and $\delta E_{2}$ through the functions $p_{\hat{2}}(x), p_{\tilde{2}}(x), \Omega^{\hat{2} \hat{3}}(x), \Omega^{\tilde{2} \tilde{3}}(x)$ defined above.

Let us consider for example the set of polarizations which belongs to the $S^{5}$. As we already have seen, all the frequencies are equal to $\Omega^{\tilde{2} \tilde{3}}(x)$. So our sum in $\delta E_{2}$ simplifies drastically and gives

$$
\delta E_{2}^{S^{5}}=4 \int_{0}^{1} \frac{d z}{\pi} \operatorname{Im}\left[\Omega^{\tilde{2} \tilde{3}}(z) \partial_{z} \log \left(1-e^{-2 i p_{\tilde{2}}(z)}\right)\right],
$$


which exactly coincides with the expression (3.6).

One can easily show that all the contributions in $\delta E_{1,2}$ can be very naturally summed up in a pretty nice way:

$$
\begin{aligned}
\delta E_{1}=2 \int_{0}^{1} \frac{d z}{\pi} \operatorname{Im}\left(p_{\hat{2}}-p_{\tilde{2}}\right) \partial_{z} \operatorname{Im}\left(\Omega^{\hat{2} \hat{3}}-\Omega^{\tilde{2} \tilde{3}}\right) \\
\delta E_{2}=2 \int_{0}^{1} \frac{d z}{\pi} \operatorname{Im}\left(\partial_{z} \Omega^{\tilde{2} \tilde{3}} \log \frac{\left(1-e^{-i p_{\tilde{2}}-i \bar{p}_{\hat{2}}}\right)\left(1-e^{-i p_{\tilde{2}}+i p_{\hat{2}}}\right)}{\left(1-e^{-2 i p_{\tilde{2}}}\right)^{2}}-\right. \\
\left.\quad-\partial_{z} \Omega^{\hat{2} \hat{3}} \log \frac{\left(1-e^{-2 i p_{\hat{2}}}\right)\left(1-e^{-i p_{\hat{2}}+i \bar{p}_{\hat{2}}}\right)}{\left(1-e^{-i p_{\hat{2}}-i p_{\tilde{2}}}\right)^{2}}\right) .
\end{aligned}
$$

Here for shortness we denote $\bar{p}(z)=p(1 / z)$.

Open Access. This article is distributed under the terms of the Creative Commons Attribution Noncommercial License which permits any noncommercial use, distribution, and reproduction in any medium, provided the original author(s) and source are credited.

\section{References}

[1] J.M. Maldacena, The large- $N$ limit of superconformal field theories and supergravity, Int. J. Theor. Phys. 38 (1999) 1113 [Adv. Theor. Math. Phys. 2 (1998) 231] [hep-th/9711200] [SPIRES].

[2] E. Witten, Anti-de Sitter space and holography, Adv. Theor. Math. Phys. 2 (1998) 253 [hep-th/9802150] [SPIRES].

[3] S.S. Gubser, I.R. Klebanov and A.M. Polyakov, Gauge theory correlators from non-critical string theory, Phys. Lett. B 428 (1998) 105 [hep-th/9802109] [SPIRES].

[4] L.N. Lipatov, High-energy asymptotics of multicolor QCD and exactly solvable lattice models, hep-th/9311037 [SPIRES].

[5] L.D. Faddeev and G.P. Korchemsky, High-energy QCD as a completely integrable model, Phys. Lett. B 342 (1995) 311 [hep-th/9404173] [SPIRES].

[6] J.A. Minahan and K. Zarembo, The Bethe-ansatz for $N=4$ super Yang-Mills, JHEP 03 (2003) 013 [hep-th/0212208] [SPIRES].

[7] N. Beisert, C. Kristjansen and M. Staudacher, The dilatation operator of $N=4$ super Yang-Mills theory, Nucl. Phys. B 664 (2003) 131 [hep-th/0303060] [SPIRES].

[8] I. Bena, J. Polchinski and R. Roiban, Hidden symmetries of the $A d S_{5} \times S^{5}$ superstring, Phys. Rev. D 69 (2004) 046002 [hep-th/0305116] [SPIRES].

[9] V.A. Kazakov, A. Marshakov, J.A. Minahan and K. Zarembo, Classical / quantum integrability in AdS/CFT, JHEP 05 (2004) 024 [hep-th/0402207] [SPIRES].

[10] N. Beisert et al., Review of AdS/CFT Integrability: An Overview, arXiv:1012.3982 [SPIRES]. 
[11] N. Beisert and M. Staudacher, The N=4 SYM Integrable Super Spin Chain, Nucl. Phys. B 670 (2003) 439 [hep-th/0307042] [SPIRES].

[12] N. Beisert and M. Staudacher, Long-range PSU $(2,2 \mid 4)$ Bethe ansaetze for gauge theory and strings, Nucl. Phys. B 727 (2005) 1 [hep-th/0504190] [SPIRES].

[13] N. Beisert, B. Eden and M. Staudacher, Transcendentality and crossing, J. Stat. Mech. (2007) P01021 [hep-th/0610251] [SPIRES].

[14] N. Gromov, V. Kazakov and P. Vieira, Exact Spectrum of Anomalous Dimensions of Planar $N=4$ Supersymmetric Yang-Mills Theory, Phys. Rev. Lett. 103 (2009) 131601 [arXiv:0901.3753] [SPIRES].

[15] D. Bombardelli, D. Fioravanti and R. Tateo, Thermodynamic Bethe Ansatz for planar AdS/CFT: a proposal, J. Phys. A 42 (2009) 375401 [arXiv:0902.3930] [SPIRES].

[16] N. Gromov, V. Kazakov, A. Kozak and P. Vieira, Exact Spectrum of Anomalous Dimensions of Planar $N=4$ Supersymmetric Yang-Mills Theory: TBA and excited states, Lett. Math. Phys. 91 (2010) 265 [arXiv:0902.4458] [SPIRES].

[17] G. Arutyunov and S. Frolov, Thermodynamic Bethe Ansatz for the AdS $S_{5} \times S^{5}$ Mirror Model, JHEP 05 (2009) 068 [arXiv:0903.0141] [SPIRES].

[18] A. Cavaglia, D. Fioravanti and R. Tateo, Extended Y-system for the $A d S_{5} / C F T_{4}$ correspondence, Nucl. Phys. B 843 (2011) 302 [arXiv: 1005.3016] [SPIRES].

[19] N. Gromov, Y-system and Quasi-Classical Strings, JHEP 01 (2010) 112 [arXiv:0910.3608] [SPIRES].

[20] N. Gromov, V. Kazakov and Z. Tsuboi, PSU $(2,2 \mid 4)$ Character of Quasiclassical AdS/CFT, JHEP 07 (2010) 097 [arXiv: 1002.3981] [SPIRES].

[21] F. Fiamberti, A. Santambrogio, C. Sieg and D. Zanon, Wrapping at four loops in $N=4$ SYM, Phys. Lett. B 666 (2008) 100 [arXiv:0712.3522] [SPIRES].

[22] V.N. Velizhanin, The four-loop anomalous dimension of the Konishi operator in $N=4$ supersymmetric Yang-Mills theory, JETP Lett. 89 (2009) 6 [arXiv:0808.3832] [SPIRES].

[23] G. Arutyunov, S. Frolov and R. Suzuki, Five-loop Konishi from the Mirror TBA, JHEP 04 (2010) 069 [arXiv: 1002.1711] [SPIRES].

[24] J. Balog and A. Hegedus, 5-loop Konishi from linearized TBA and the XXX magnet, JHEP 06 (2010) 080 [arXiv: 1002.4142] [SPIRES].

[25] J. Balog and A. Hegedus, The Bajnok-Janik formula and wrapping corrections, JHEP 09 (2010) 107 [arXiv: 1003.4303] [SPIRES].

[26] Z. Bajnok and R.A. Janik, Four-loop perturbative Konishi from strings and finite size effects for multiparticle states, Nucl. Phys. B 807 (2009) 625 [arXiv:0807.0399] [SPIRES].

[27] Z. Bajnok, A. Hegedus, R.A. Janik and T. Lukowski, Five loop Konishi from AdS/CFT, Nucl. Phys. B $\mathbf{8 2 7}$ (2010) 426 [arXiv:0906.4062] [SPIRES].

[28] A.V. Kotikov, L.N. Lipatov, A. Rej, M. Staudacher and V.N. Velizhanin, Dressing and Wrapping, J. Stat. Mech. (2007) P10003 [arXiv:0704.3586] [SPIRES].

[29] Z. Bajnok, R.A. Janik and T. Lukowski, Four loop twist two, BFKL, wrapping and strings, Nucl. Phys. B 816 (2009) 376 [arXiv:0811.4448] [SPIRES]. 
[30] T. Lukowski, A. Rej and V.N. Velizhanin, Five-Loop Anomalous Dimension of Twist-Two Operators, Nucl. Phys. B 831 (2010) 105 [arXiv:0912.1624] [SPIRES].

[31] A.V. Kotikov and L.N. Lipatov, DGLAP and BFKL evolution equations in the $N=4$ supersymmetric gauge theory, Nucl. Phys. B 661 (2003) 19 [hep-ph/0208220] [SPIRES].

[32] N. Gromov, V. Kazakov and P. Vieira, Exact Spectrum of Planar $\mathcal{N}=4$ Supersymmetric Yang-Mills Theory: Konishi Dimension at Any Coupling, Phys. Rev. Lett. 104 (2010) 211601 [arXiv:0906.4240] [SPIRES].

[33] S. Frolov, Konishi operator at intermediate coupling, J. Phys. A 44 (2011) 065401 [arXiv: 1006.5032] [SPIRES].

[34] S.S. Gubser, I.R. Klebanov and A.M. Polyakov, A semi-classical limit of the gauge/string correspondence, Nucl. Phys. B 636 (2002) 99 [hep-th/0204051] [SPIRES].

[35] F. Passerini, J. Plefka, G.W. Semenoff and D. Young, On the Spectrum of the AdS $S_{5} \times S^{5}$ String at large lambda, JHEP 03 (2011) 046 [arXiv: 1012.4471] [SPIRES].

[36] R. Roiban and A.A. Tseytlin, Quantum strings in $A d S_{5} \times S^{5}$ : strong-coupling corrections to dimension of Konishi operator, JHEP 11 (2009) 013 [arXiv: 0906.4294] [SPIRES].

[37] G. Arutyunov, S. Frolov and R. Suzuki, Exploring the mirror TBA, JHEP 05 (2010) 031 [arXiv: 0911.2224] [SPIRES].

[38] S. Schäfer-Nameki, Review of AdS/CFT Integrability, Chapter II.4: The Spectral Curve, arXiv: 1012.3989 [SPIRES].

[39] N. Gromov, V. Kazakov, S. Leurent and D. Volin, work in progress.

[40] S. Frolov, A. Tirziu and A.A. Tseytlin, Logarithmic corrections to higher twist scaling at strong coupling from AdS/CFT, Nucl. Phys. B 766 (2007) 232 [hep-th/0611269] [SPIRES].

[41] P.Y. Casteill and C. Kristjansen, The Strong Coupling Limit of the Scaling Function from the Quantum String Bethe Ansatz, Nucl. Phys. B 785 (2007) 1 [arXiv:0705.0890] [SPIRES].

[42] A.V. Belitsky, Strong coupling expansion of Baxter equation in $N=4$ SYM, Phys. Lett. B 659 (2008) 732 [arXiv:0710.2294] [SPIRES].

[43] N. Gromov, Generalized Scaling Function at Strong Coupling, JHEP 11 (2008) 085 [arXiv:0805.4615] [SPIRES].

[44] D.J. Gross and F. Wilczek, Asymptotically free gauge theories. 2, Phys. Rev. D 9 (1974) 980 [SPIRES].

[45] G.P. Korchemsky, Asymptotics of the Altarelli-Parisi-Lipatov Evolution Kernels of Parton Distributions, Mod. Phys. Lett. A 4 (1989) 1257 [SPIRES].

[46] G.P. Korchemsky and G. Marchesini, Structure function for large $x$ and renormalization of Wilson loop, Nucl. Phys. B 406 (1993) 225 [hep-ph/9210281] [SPIRES].

[47] A.V. Belitsky, A.S. Gorsky and G.P. Korchemsky, Logarithmic scaling in gauge/string correspondence, Nucl. Phys. B $\mathbf{7 4 8}$ (2006) 24 [hep-th/0601112] [SPIRES].

[48] D. Volin, The 2-loop generalized scaling function from the BES/FRS equation, arXiv: 0812.4407 [SPIRES].

[49] S. Giombi, R. Ricci, R. Roiban, A.A. Tseytlin and C. Vergu, Generalized scaling function from light-cone gauge $A d S_{5} \times S^{5}$ superstring, JHEP 06 (2010) 060 [arXiv: 1002.0018] [SPIRES]. 
[50] A.V. Kotikov and L.N. Lipatov, On the highest transcendentality in $N=4$ SUSY, Nucl. Phys. B 769 (2007) 217 [hep-th/0611204] [SPIRES].

[51] M.K. Benna, S. Benvenuti, I.R. Klebanov and A. Scardicchio, A test of the AdS/CFT correspondence using high-spin operators, Phys. Rev. Lett. 98 (2007) 131603 [hep-th/0611135] [SPIRES].

[52] L.F. Alday, G. Arutyunov, M.K. Benna, B. Eden and I.R. Klebanov, On the strong coupling scaling dimension of high spin operators, JHEP 04 (2007) 082 [hep-th/0702028] [SPIRES].

[53] I. Kostov, D. Serban and D. Volin, Strong coupling limit of Bethe ansatz equations, Nucl. Phys. B 789 (2008) 413 [hep-th/0703031] [SPIRES].

[54] M. Beccaria, G.F. De Angelis and V. Forini, The scaling function at strong coupling from the quantum string Bethe equations, JHEP 04 (2007) 066 [hep-th/0703131] [SPIRES].

[55] B. Basso, G.P. Korchemsky and J. Kotanski, Cusp anomalous dimension in maximally supersymmetric Yang-Mills theory at strong coupling, Phys. Rev. Lett. 100 (2008) 091601 [arXiv: 0708.3933] [SPIRES].

[56] I. Kostov, D. Serban and D. Volin, Functional BES equation, JHEP 08 (2008) 101 [arXiv:0801.2542] [SPIRES].

[57] B. Eden and M. Staudacher, Integrability and transcendentality, J. Stat. Mech. (2006) P11014 [hep-th/0603157] [SPIRES].

[58] L. Freyhult, Review of AdS/CFT Integrability, Chapter III.4: Twist states and the cusp anomalous dimension, arXiv:1012.3993 [SPIRES].

[59] L. Freyhult and S. Zieme, The virtual scaling function of AdS/CFT, Phys. Rev. D 79 (2009) 105009 [arXiv:0901.2749] [SPIRES].

[60] D. Fioravanti, P. Grinza and M. Rossi, Beyond cusp anomalous dimension from integrability, Phys. Lett. B 675 (2009) 137 [arXiv:0901.3161] [SPIRES].

[61] S. Schäfer-Nameki and M. Zamaklar, Stringy sums and corrections to the quantum string Bethe ansatz, JHEP 10 (2005) 044 [hep-th/0509096] [SPIRES].

[62] M. Beccaria, G.V. Dunne, V. Forini, M. Pawellek and A.A. Tseytlin, Exact computation of one-loop correction to energy of spinning folded string in $A d S_{5} \times S^{5}$,

J. Phys. A 43 (2010) 165402 [arXiv:1001.4018] [SPIRES].

[63] S. Giombi, R. Ricci, R. Roiban and A.A. Tseytlin, Two-loop $A d S_{5} \times S^{5}$ superstring: testing asymptotic Bethe ansatz and finite size corrections, J. Phys. A 44 (2011) 045402 [arXiv: 1010.4594] [SPIRES].

[64] B. Basso and G.P. Korchemsky, Anomalous dimensions of high-spin operators beyond the leading order, Nucl. Phys. B 775 (2007) 1 [hep-th/0612247] [SPIRES].

[65] D. Fioravanti, P. Grinza and M. Rossi, On the logarithmic powers of $\operatorname{sl}(2) S Y M_{4}$, Phys. Lett. B 684 (2010) 52 [arXiv:0911.2425] [SPIRES].

[66] L.F. Alday and J.M. Maldacena, Comments on operators with large spin, JHEP 11 (2007) 019 [arXiv:0708.0672] [SPIRES].

[67] B. Basso and G.P. Korchemsky, Embedding nonlinear $O(6) \sigma$-model into $N=4$ super-YangMills theory, Nucl. Phys. B 807 (2009) 397 [arXiv: 0805.4194] [SPIRES].

[68] D. Fioravanti, P. Grinza and M. Rossi, Strong coupling for planar $\mathcal{N}=4$ SYM theory: an all-order result, Nucl. Phys. B 810 (2009) 563 [arXiv:0804.2893] [SPIRES]. 
[69] B. Basso, to appear.

[70] N. Gromov and P. Vieira, The $A d S_{5} \times S^{5}$ superstring quantum spectrum from the algebraic curve, Nucl. Phys. B 789 (2008) 175 [hep-th/0703191] [SPIRES].

[71] V. Mikhaylov, On the Fermionic Frequencies of Circular Strings, J. Phys. A 43 (2010) 335401 [arXiv:1002.1831] [SPIRES].

[72] N. Beisert, S. Frolov, M. Staudacher and A.A. Tseytlin, Precision spectroscopy of AdS/CFT, JHEP 10 (2003) 037 [hep-th/0308117] [SPIRES].

[73] V.A. Kazakov and K. Zarembo, Classical/quantum integrability in non-compact sector of AdS/CFT, JHEP 10 (2004) 060 [hep-th/0410105] [SPIRES].

[74] N. Beisert, V.A. Kazakov, K. Sakai and K. Zarembo, The algebraic curve of classical superstrings on $A d S_{5} \times S^{5}$, Commun. Math. Phys. 263 (2006) 659 [hep-th/0502226] [SPIRES].

[75] N. Gromov, S. Schäfer-Nameki and P. Vieira, Efficient precision quantization in AdS/CFT, JHEP 12 (2008) 013 [arXiv:0807.4752] [SPIRES].

[76] N. Gromov and P. Vieira, Constructing the AdS/CFT dressing factor, Nucl. Phys. B 790 (2008) 72 [hep-th/0703266] [SPIRES].

[77] A. Rej and F. Spill, Konishi at strong coupling from ABE, J. Phys. A 42 (2009) 442003 [arXiv:0907.1919] [SPIRES].

[78] V. Kazakov and N. Gromov, Why Y? Exploiting Hirota integrable dynamics in AdS/CFT, Talk at IGST-2010, Nordita, Stockholm, http://agenda.albanova.se/contributionDisplay.py?contribId=258\&confId=1561.

[79] S. Frolov, Scaling dimensions from the mirror TBA, Talk at QFT2010, DESY, Hamburg, http://qft2010.desy.de/sites/site_qft2010/content/e88901/e104143/infoboxContent104215/ Frolov_DESY10.pdf.

[80] M. Beccaria, V. Forini, A. Tirziu and A.A. Tseytlin, Structure of large spin expansion of anomalous dimensions at strong coupling, Nucl. Phys. B 812 (2009) 144 [arXiv: 0809.5234] [SPIRES].

[81] D. Fioravanti, P. Grinza and M. Rossi, Beyond cusp anomalous dimension from integrability in $S Y M_{4}$, AIP Conf. Proc. 1350 (2011) 233 [arXiv:1011.4005] [SPIRES].

[82] G.P. Korchemsky, Quasiclassical QCD Pomeron, Nucl. Phys. B 462 (1996) 333 [hep-th/9508025] [SPIRES].

[83] R. Roiban and A.A. Tseytlin, Semiclassical string computation of strong-coupling corrections to dimensions of operators in Konishi multiplet, Nucl. Phys. B 848 (2011) 251 [arXiv:1102.1209] [SPIRES].

[84] B.C. Vallilo and L. Mazzucato, The Konishi multiplet at strong coupling, arXiv:1102.1219 [SPIRES]. 